\title{
BMJ Open Modified minimally invasive surgical technique plus Bio-Oss Collagen for regenerative therapy of isolated interdental intrabony defects: study protocol for a randomised controlled trial
}

Churen Zhang (D) , Haidong Zhang, Zhaoguo Yue, Lili Miao, Ye Han, Kaining Liu, Jianxia Hou

To cite: Zhang $\mathrm{C}$, Zhang $\mathrm{H}$, Yue Z, et al. Modified minimally invasive surgical technique plus Bio-0ss Collagen for regenerative therapy of isolated interdental intrabony defects: study protocol for a randomised controlled trial. BMJ Open 2020;10:e040046. doi:10.1136/ bmjopen-2020-040046

- Prepublication history and additional material for this paper is available online. To view these files, please visit the journal online (http://dx.doi.org/10. 1136/bmjopen-2020-040046).

Received 04 May 2020 Revised 09 September 2020 Accepted 23 September 2020

\section{Check for updates}

(C) Author(s) (or their employer(s)) 2020. Re-use permitted under CC BY-NC. No commercial re-use. See rights and permissions. Published by BMJ.

Department of Periodontology, Peking University School and Hospital of Stomatology and National Engineering Laboratory for Digital and Material Technology of Stomatology and Beijing Key Laboratory of Digital Stomatology, Beijing, China

Correspondence to Dr Kaining Liu; liukainingbjmu@163.com and Professor Jianxia Hou; jxhou@163.com

\section{ABSTRACT}

Introduction Periodontal regeneration surgery has been widely used to deal with intrabony defects. Modified minimally invasive surgical technique (M-MIST) is designed to deal with isolated interdental intrabony defects, and has achieved satisfactory periodontal regenerative effect. Bio-Oss Collagen, as a bioactive material, has been applied for periodontal regeneration. It is similar to human cancellous bone, with the ability to promote bone formation; furthermore, it has exceptional plasticity and spatial stability. The combination of different materials and techniques has become a research hotspot in recent years. By combining the superiority of regeneration technology and materials, better regenerative effect can be achieved. This study will search for differences between M-MIST combined with Bio-0ss Collagen, and M-MIST alone in regeneration therapy for intrabony defects.

Methods and analysis The present research is designed as a two-group parallel randomised controlled trial. The total number of patients is 40 . The patients will be randomly assigned to two groups, with 20 participants in each group, for further periodontal regenerative surgery. Test group: $\mathrm{M}$ MIST plus Bio-Oss Collagen. Control group: M-MIST. After 12 months, the measurement indices will be recorded; these will include clinical attachment gain and radiographical intrabony defect depth change as the primary results, and secondary outcomes of full-mouth plaque scores, probing depth, fullmouth bleeding scores, gingival recession, mobility, gingival papilla height and Visual Analogue Scale. The paired samples $\mathrm{t}$-test will be applied to detect any difference between baseline and 1-year registrations. A general linear model will be performed to study the relationship between the secondary and the primary outcome.

Ethics and dissemination The present research has received approval from the Ethics Committee of Peking University School and Hospital of Stomatology (PKUSSIRB-202053002). Data of the present research will be registered with the International Clinical Trials Registry Platform. Additionally, we will disseminate the results through scientific dental journals.
Strengths and limitations of this study

- This trial is designed as a randomised, examinerblind clinical trial.

- The trial will be the first clinical study to compare the periodontal regenerative effect of modified minimally invasive surgical technique (M-MIST) plus Bio-0ss Collagen and M-MIST exclusively in isolated interdental intrabony defects.

- The follow-up in the trial will last for 1 year.

- The outcome of this study will not be applied to patients with systemic disease.

Trial registration number ChiCTR-2000030851. Protocol version Protocol Version 4, 14 July 2020.

\section{INTRODUCTION}

Periodontal disease is an inflammatory disease, with a high incidence around the teeth. It can lead to loss of periodontal attachment and alveolar bone, and even to tooth loss. ${ }^{12}$ Periodontal disease is caused by multiple factors, of which the initial factor is the interaction between the biofilm and the immune response. ${ }^{3}$ Periodontal pathogens play a key role in the pathogenesis and development of periodontal and systemic diseases, including diabetes and cardiovascular diseases, which can influence the development of periodontal disease. ${ }^{4}$ It is reported that periodontitis is one of the most common diseases around the world. ${ }^{5}$ Scaling and root planning (SRP) is the essential therapy for every patient. ${ }^{6}$ However, for teeth with deep periodontal pockets, SRP cannot achieve a better therapeutic effect than surgery. ${ }^{7}$ After periodontal initial therapy, the residual 
deep pockets have often been associated with intrabony defects, identified as a clinical challenge. ${ }^{8}$ The intrabony defects may result in unmanageable inflammation, and ultimately to tooth loss. ${ }^{9}$ Therefore, intrabony defects are generally regarded as surgical indications. ${ }^{10}$

Periodontal regeneration surgery has been widely applied to deal with intrabony defects by reforming the periodontal attachment in the hopes of saving the involved teeth. ${ }^{11-14}$ In the past 20 years, the development of periodontal regeneration therapy has been reflected mainly in two aspects. First, surgical design and techniques have been studied, especially minimally invasive surgery. ${ }^{15-20}$ Second, considerable progress has been made in regenerative materials, such as enamel matrix derivative (EMD),${ }^{18}$ demineralised freeze-dried bone allograft, ${ }^{19}$ recombinant human platelet-derived growth factor BB $(\text { rhPDGF-BB })^{20}$ and spongy bone with collagen (Bio-Oss Collagen). ${ }^{21}$ These methods offer more advantages than simple flap debridement in increasing clinical attachment and decreasing the probing depth of the affected teeth. ${ }^{22}{ }^{23}$ However, common complications, such as the exposure of barrier membrane and embedded material, have contributed to poor clinical results of periodontal regeneration surgery. ${ }^{24}$

To solve this problem, many periodontal surgical designs and techniques have been proposed and continually improved on. ${ }^{25-28}$ As early as in 1995, Harrel and Ress $^{25}$ suggested applying minimally invasive surgery to periodontal surgical treatment, whose key points were small incision, small flap and reduction of damage to soft and hard tissues. In 1995 and 1999, Cortellini et $a l^{2627}$ proposed papilla preservation techniques to preserve interdental soft tissues as completely as possible and isolate the operative area from the oral environment. Based on this, to further improve the surgical effect, the concept of minimally invasive surgery was proposed and gradually improved on. In 2007, Cortellini and Tonetti ${ }^{28}$ proposed a minimally invasive surgical technique (MIST) to deal with periodontal intrabony defects for more periodontal tissue regeneration. This technique was designed to reduce surgical trauma, operative time and postoperative discomfort. ${ }^{28} \mathrm{~A}$ number of clinical studies ${ }^{162}$ have confirmed the effectiveness and advantages of the technique. In 2009, Cortellini and Tonetti ${ }^{17}$ further designed a modified MIST (M-MIST). This method only opened the tiny buccal flap to ensure adequate blood supply, tighter primary wound closure and lower risk of bacterial infection.

In recent years, the combination of different materials and techniques has become a research hotspot. ${ }^{30}$ By combining the advantages of regeneration technology and materials, better regenerative results can be achieved. ${ }^{31}$ M-MIST only opens a small flap on the buccal side to achieve minimal interdental passage, saving the palatal soft tissue. ${ }^{17}$ Bio-Oss Collagen is similar to human cancellous bone, with the ability to promote bone formation. ${ }^{32}$ Bio-Oss Collagen, with outstanding plasticity and spatial stability, ${ }^{33}$ is suitable for the small field of vision resulting from M-MIST. Therefore, we plan to research the therapeutic effect of M-MIST combined with Bio-Oss Collagen and the use of M-MIST alone for periodontal tissue regeneration of isolated interdental intrabony defects. The presumption was raised that combining M-MIST and Bio-Oss Collagen would lead to a better result in periodontally regenerating intrabony defects than M-MIST alone. The practicability, applicability and extensibility of the combination will be investigated in the present study. The primary outcomes were clinical attachment gain and radiographical bone-filling of the intrabony defect, which would be recorded by clinical examination, periapical radiographs and cone beam CT. The clinical attachment gain and bone filling represent periodontal tissue regeneration. ${ }^{17}$ The secondary outcomes were probing depth, full-mouth plaque scores, full-mouth bleeding scores, gingival recession, mobility, height of the gingival papilla and Visual Analogue Scale (VAS). These indices represent the inflammatory characteristics of periodontal tissue. ${ }^{28}$ The present article describes the design of the trial.

\section{METHODS AND ANALYSIS}

This research is a randomised controlled trial with two parallel groups. It will be carried out according to the World Medical Association's Declaration of Helsinki. Patients with stage III or IV periodontitis ${ }^{34}$ (details in the online supplemental material 1) in need of periodontal regenerative treatment for isolated intrabony defects are the potential subjects. This study will be undertaken at the Peking University School and Hospital of Stomatology (Beijing, China). The present research has received approval from the Ethics Committee of Peking University School and Hospital of Stomatology (PKUSSIRB-202053002). The research framework is shown in figure 1 .

\section{Participant selection}

All participants will come from the Periodontology Department of Peking University School and Hospital of Stomatology. At re-evaluation after periodontal initial therapy, if the patient is found to have isolated intrabony defects, he or she will be informed about the study. The potential patients will receive information about the research. The patients will be incorporated into this trial only after their signature has been obtained (informed consent in the online supplemental material 2). The personal information of the consent form will be confidentially stored in our department.

As regards patient characteristics, the following criteria should be met: (1) age 18-75 years; (2) both genders will be considered for selection in the study; (3) patients with stage III or IV periodontitis at least 2 months after periodontal initial therapy; (4) good compliance; (5) good oral hygiene; (6) full-mouth plaque score and full-mouth bleeding score, each less than $30 \%$; and (7) systemically healthy. The intrabony defect should be an isolated 


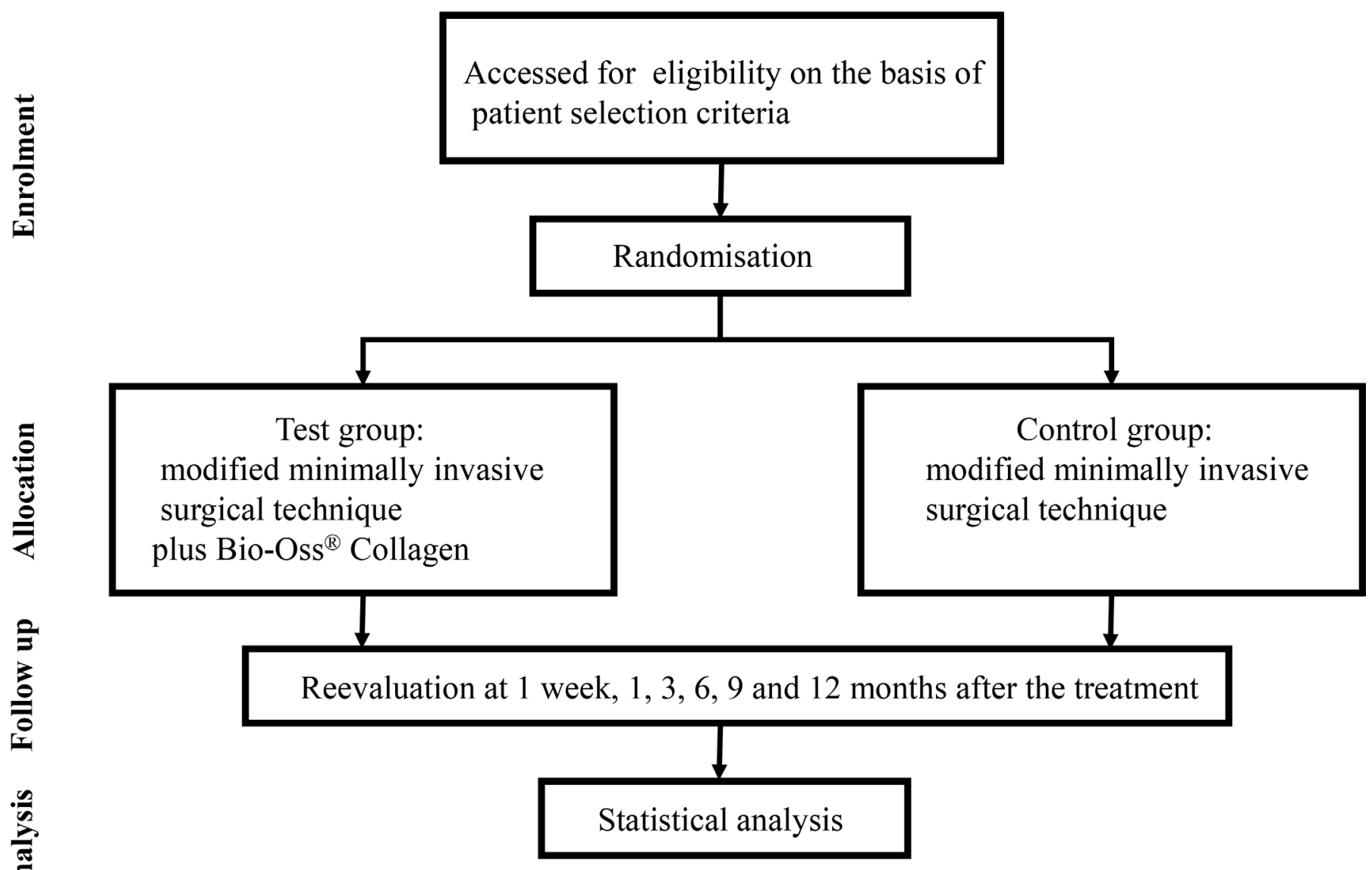

Figure 1 Framework of the trial and Consolidated Standards of Reporting Trials flow chart.

intrabony defect of more than $3 \mathrm{~mm}$ depth, combined with more than $5 \mathrm{~mm}$ probing depth and attachment loss. In addition, the area of the intrabony defect should not exceed the lingual surface area of the root. The morphology of the intrabony defect will be detected during the operation and finally determined whether the patient would be enrolled in the trial. The associated tooth should either maintain normal pulp vitality or should have undergone root canal therapy for at least 6 months. The intrabony defect existing around the anterior teeth, the premolars or at the mesial side of mandibular first molars will be included to avoid the influence of furcation involvement. Patients with tumours, systemic diseases or a history of receiving antibiotics in the past 3 months will be excluded. Patients having affected teeth with $3^{\circ}$ mobility, furcation involvement, acute periapical inflammation or root fractures will not be enrolled.

\section{Randomisation and blinding}

The random sequence is produced through a random number table and the assignment is saved in a sealed envelope. A research worker unaware of the trial process will be in charge of the randomisation. All patients will be randomly assigned to two groups. Test group: M-MIST combined with Bio-Oss Collagen. Control group: M-MIST. All surgical operations will be performed by an experienced therapist who is unaware of the assignment.
Another two members who are unaware of the research plan will respectively take charge of the clinical examination and statistical analysis.

\section{Interventions}

The enrolled participants will receive a periodontal clinical examination. They will have periapical radiographs and cone beam CT taken. The surgical process will be performed as described in the literature. ${ }^{17}$ If the width of the top of the gingival papilla is $2 \mathrm{~mm}$ or narrower, the simplified papilla preservation flap ${ }^{27}$ will be performed; if it is wider than $2 \mathrm{~mm}$, the modified papilla preservation technique $^{26}$ will be used. Then the incision will extend along the gingival sulcus on the buccal side of the two adjacent teeth, and a buccal flap will be reflected to expose $1-2 \mathrm{~mm}$ of buccal bone crest. With miniature blades and mini curettes, the granulation tissue will be curetted and the roots will be carefully planned with curettes. If the depth of the intrabony defect is $3 \mathrm{~mm}$ or more and the defect does not contain a lingual intrabony component, the site will be finally enrolled for further examination and statistical analysis. If the intrabony defects do not meet the inclusion criteria, the surgery will be continued, but such defects will not be enrolled for further examination or statistical analysis. After thorough debridement and careful rinsing with saline solution, the intrabony defect will be filled with Bio-Oss Collagen to the level of 
the buccal bone crest in the test group. In the control group, intrabony defects will not be treated with any other materials. Finally, a vertical mattress suture will be performed to close the wound. Periapical films of the site associated with the defect will be taken immediately after the surgery. If the tooth appears to have $2^{\circ}$ to $3^{\circ}$ mobility after the surgery, the tooth will be promptly splinted. The patients will be required to rinse with a $0.2 \%$ chlorhexidine solution for 1 week, and the suture will be removed 1 week after surgery.

The surgeries will be performed at the Periodontology Department, Peking University School and Hospital of Stomatology. An experienced periodontist with the help of an operating microscope will perform all the operations.

\section{Examination}

At baseline, all the enrolled patients will receive a periodontal examination by two experienced research professionals who have passed the inter-examiner agreement exam. Relative periodontal indexes will include fullmouth plaque score ${ }^{28}$ clinical attachment loss of the involved teeth, ${ }^{29}$ full-mouth bleeding score, ${ }^{28}$ gingival recession of the involved teeth, ${ }^{14}$ mobility of the involved teeth, ${ }^{28}$ probing depth of the involved teeth,${ }^{29}$ height of the gingival papilla, ${ }^{29}$ periapical films and cone beam CT of the interdental site. ${ }^{28}$ During the surgery, the defect anatomy, including the depth and number of walls of the intrabony defects, will be examined by the operator. The patients will receive a phone call for re-examination at 1 week, and 1, 3, 6, 9, and 12 months post surgery. At 1 , $3,6,9$ and 12 months post surgery, a full-mouth plaque score and gingival papilla height will be recorded by the same calibrated examiners. At 12 months post treatment, a full-mouth plaque score, clinical attachment gain of the involved teeth, gingival recession, mobility, full-mouth bleeding score, gingival papilla height and probing depth of the involved teeth will be examined by the same examiners. Periapical films and cone beam CT of the site associated with the defect will be taken 6 and 12 months post surgery. In addition, patients will finish a VAS to evaluate the discomfort after 1 week of treatment. During the follow-up, complications will be recorded and treated once they happen. At re-evaluation, if the oral hygiene is deficient, we will clean the teeth by supragingival scaling and reinforcing oral hygiene instruction. All the data will be recorded in the periodontal examination charts, and registered and stored in the computer. There is no data monitoring committee in our hospital, so to ensure the correction and integrity of data, two different researchers will take charge of the management and storage of data.

\section{Sample size}

The needed number of participants was calculated according to the formula:

$$
\mathrm{n} 1=\mathrm{n} 2=2\left[\frac{\sigma\left(\mathrm{Z}_{\alpha / 2}+\mathrm{Z}_{\beta}\right)}{\delta}\right]^{2}
$$

On the basis of previous literature, ${ }^{1735}$ the difference of clinical attachment gain using Bio-Oss Collagen or not using it $(\delta)$ was about $1.5 \mathrm{~mm}$ and the $\mathrm{SD}(\sigma)$ was about $1.4 \mathrm{~mm}$. The power of test $(\beta)$ is set as $10 \%$ and the inspection level $(\alpha)$ is set as 0.05 . After calculation, 18 participants will be needed for each group. Assuming that the rate of participants lost during follow-up is around $10 \%, 20$ participants will be needed for each group. Ultimately, at least 40 participants will be needed in all.

\section{Statistical analysis}

One patient will be identified as a data unit. If two or more intrabony defects go through the surgery in one patient, only one defect nearest to the midline will be enrolled. ${ }^{36}$ The paired samples t-test will be applied to detect any difference between baseline and 1-year registrations. A general linear model will be performed to study the relationship between the defect depth, number of bony walls, full-mouth plaque score and full-mouth bleeding score with clinical attachment gain, probing depth reduction and radiographical intrabony defect depth change. The level of statistically significant difference will be set at 0.05 . Statistical analysis will be performed using SPSS V.26.0 software.

\section{Withdrawal}

Participants will be told that they can quit the research at any time. The withdrawal will not influence their receiving help from departmental periodontists in the future.

\section{Dissemination of data}

Data from the present research will be registered with the International Clinical Trials Registry Platform. Additionally, we will disseminate the results through scientific dental journals.

\section{DISCUSSION}

For an isolated intrabony defect, M-MIST could be an effective treatment method, with an average clinical attachment gain of $4.8 \mathrm{~mm}$ at 1 year post surgery. ${ }^{17} \mathrm{It}$ allows access to the root surface with only the buccal flap opening, which is minimally invasive and further enhances wound stability. However, when bioactive materials, such as EMD and rhPDGF-BB, were used in combination with M-MIST, periodontal regenerative effects were not better. ${ }^{3738}$ EMD might not be an ideal bioactive material to deal with wide defects. ${ }^{39}$ Bio-Oss Collagen is a bovinederived xenograft containing profuse collagen that can fit into different types of defects. ${ }^{33}$ The present research is designed to study the effectiveness and potential applicability of M-MIST combined with Bio-Oss Collagen for tissue regeneration of periodontal intrabony defects. If the results of M-MIST combined with Bio-Oss Collagen turned out to be better than those of M-MIST alone in terms of radiographical and clinical defect improvement, Bio-Oss Collagen might be considered for application in 
combination with M-MIST for periodontal tissue regeneration of intrabony defects.

\section{Ethics and dissemination}

The present research has received approval from the Ethics Committee of Peking University School and Hospital of Stomatology (PKUSSIRB-202053002). The patients will be incorporated into this trial only after their signature has been obtained. The study will be performed according to the 2013 revision of the Helsinki Declaration of 1975. Personal information of all subjects will be stored in the Department of Periodontology of Peking University School and Hospital of Stomatology. Data of the present research will be registered with the International Clinical Trials Registry Platform. Additionally, we will disseminate the results through scientific journals.

\section{Trial status}

The trial protocol received ethics approval in March 2020 and was registered at the International Clinical Trials Registry Platform on 15 March 2020. The trial will begin after the COVID-19 pandemic has been controlled; it is scheduled to be completed in October 2021.

Contributors $\mathrm{HZ}, \mathrm{KL}, \mathrm{CZ}$ and $\mathrm{JH}$ proposed the concept of this work. $\mathrm{CZ}, \mathrm{KL}$ and $\mathrm{JH}$ designed the trial. $\mathrm{CZ}$ and $\mathrm{HZ}$ drafted the document. ZY, LM and YH revised the part on randomisation and calculation of the sample size. KL and JH reviewed and finalised the manuscript. All authors agreed with the final version.

Funding Supported by the Peking University School and Hospital of Stomatology grant PKUSSNCT-20A04

Competing interests None declared.

Patient consent for publication Not required.

Provenance and peer review Not commissioned; externally peer-reviewed.

Supplemental material This content has been supplied by the author(s). It has not been vetted by BMJ Publishing Group Limited (BMJ) and may not have been peer-reviewed. Any opinions or recommendations discussed are solely those of the author(s) and are not endorsed by BMJ. BMJ disclaims all liability and responsibility arising from any reliance placed on the content. Where the content includes any translated material, BMJ does not warrant the accuracy and reliability of the translations (including but not limited to local regulations, clinical guidelines, terminology, drug names and drug dosages), and is not responsible for any error and/or omissions arising from translation and adaptation or otherwise.

Open access This is an open access article distributed in accordance with the Creative Commons Attribution Non Commercial (CC BY-NC 4.0) license, which permits others to distribute, remix, adapt, build upon this work non-commercially, and license their derivative works on different terms, provided the original work is properly cited, appropriate credit is given, any changes made indicated, and the use is non-commercial. See: http://creativecommons.org/licenses/by-nc/4.0/.

ORCID iD

Churen Zhang http://orcid.org/0000-0001-9070-7138

\section{REFERENCES}

1 Tonetti MS, Eickholz P, Loos BG, et al. Principles in prevention of periodontal diseases: consensus report of group 1 of the 11th European workshop on periodontology on effective prevention of periodontal and peri-implant diseases. J Clin Periodontol 2015;42 Suppl 16:S5-11.

2 Ramseier CA, Anerud A, Dulac M, et al. Natural history of periodontitis: Disease progression and tooth loss over 40 years. $J$ Clin Periodontol 2017;44:1182-91.

3 Meyle J, Chapple I. Molecular aspects of the pathogenesis of periodontitis. Periodontol 2000 2015;69:7-17.
4 Hasturk $\mathrm{H}$, Kantarci A. Activation and resolution of periodontal inflammation and its systemic impact. Periodontol 2000 2015;69:255-73.

5 GBD 2015 SDG Collaborators. Measuring the health-related sustainable development goals in 188 countries: a baseline analysis from the global burden of disease study 2015. Lancet 2016;388:1813-50.

6 Berezow AB, Darveau RP. Microbial shift and periodontitis. Periodontol 2000 2011;55:36-47.

7 Serino G, Rosling B, Ramberg P, et al. Initial outcome and long-term effect of surgical and non-surgical treatment of advanced periodontal disease. J Clin Periodontol 2001;28:910-6.

8 Cortellini P, Tonetti MS. Clinical concepts for regenerative therapy in intrabony defects. Periodontol 2000 2015;68:282-307.

9 Matuliene G, Pjetursson BE, Salvi GE, et al. Influence of residual pockets on progression of periodontitis and tooth loss: results after 11 years of maintenance. $J$ Clin Periodontol 2008;35:685-95.

10 Reynolds MA, Kao RT, Camargo PM, et al. Periodontal regenerationintrabony defects: a consensus report from the AAP regeneration workshop. J Periodontol 2015;86:S105-7.

11 Sanz M, Tonetti MS, Zabalegui I, et al. Treatment of intrabony defects with enamel matrix proteins or barrier membranes: results from a multicenter practice-based clinical trial. J Periodontol 2004;75:726-33.

12 Sculean A, Kiss A, Miliauskaite A, et al. Ten-Year results following treatment of intra-bony defects with enamel matrix proteins and guided tissue regeneration. J Clin Periodontol 2008;35:817-24.

13 Paolantonio M, Femminella B, Coppolino E, et al. Autogenous periosteal barrier membranes and bone grafts in the treatment of periodontal intrabony defects of single-rooted teeth: a 12-month reentry randomized controlled clinical trial. $J$ Periodontol 2010;81:1587-95.

14 Cortellini P, Buti J, Pini Prato G, et al. Periodontal regeneration compared with access flap surgery in human intra-bony defects 20-year follow-up of a randomized clinical trial: tooth retention, periodontitis recurrence and costs. J Clin Periodontol 2017;44:58-66.

15 Wachtel H, Schenk G, Böhm S, et al. Microsurgical access flap and enamel matrix derivative for the treatment of periodontal intrabony defects: a controlled clinical study. J Clin Periodontol 2003;30:496-504.

16 Cortellini P, Tonetti MS. Minimally invasive surgical technique and enamel matrix derivative in intra-bony defects. I: clinical outcomes and morbidity. J Clin Periodontol 2007;34:1082-8.

17 Cortellini P, Tonetti MS. Improved wound stability with a modified minimally invasive surgical technique in the regenerative treatment of isolated interdental intrabony defects. J Clin Periodontol 2009;36:157-63.

18 Takeda K, Mizutani K, Matsuura T, et al. Periodontal regenerative effect of enamel matrix derivative in diabetes. PLoS One 2018;13:e0207201.

19 Sali DD, Pauline George J. Demineralized freeze dried bone allograft with amniotic membrane in the treatment of periodontal intrabony defects - 12 month randomized controlled clinical trial. J Periodontol 2016;11:1-18.

20 Li F, Yu F, Xu X, et al. Evaluation of recombinant human FGF-2 and PDGF-BB in periodontal regeneration: a systematic review and metaanalysis. Sci Rep 2017;7:65.

21 Nevins ML, Camelo M, Rebaudi A, et al. Three-dimensional microcomputed tomographic evaluation of periodontal regeneration: a human report of intrabony defects treated with bio-oss collagen. Int $J$ Periodontics Restorative Dent 2005;25:365-73.

22 Aimetti M, Romano F, Pigella E, et al. Treatment of wide, shallow, and predominantly 1 -wall intrabony defects with a bioabsorbable membrane: a randomized controlled clinical trial. $J$ Periodontol 2005;76:1354-61.

23 Nickles K, Ratka-Krüger P, Neukranz E, et al. Open flap debridement and guided tissue regeneration after 10 years in infrabony defects. J Clin Periodontol 2009;36:976-83.

24 Graziani F, Gennai S, Cei S, et al. Clinical performance of access flap surgery in the treatment of the intrabony defect. A systematic review and meta-analysis of randomized clinical trials. J Clin Periodontol 2012;39:145-56.

25 Harrel SK, Rees TD. Granulation tissue removal in routine and minimally invasive procedures. Compend Contin Educ Dent 1995;16:960-2.

26 Cortellini P, Prato GP, Tonetti MS. The modified papilla preservation technique. A new surgical approach for interproximal regenerative procedures. J Periodontol 1995;66:261-6.

27 Cortellini P, Prato GP, Tonetti MS. The simplified papilla preservation flap. A novel surgical approach for the management of soft tissues in regenerative procedures. Int $J$ Periodontics Restorative Dent 1999;19:589-99. 
28 Cortellini P, Tonetti MS. A minimally invasive surgical technique with an enamel matrix derivative in the regenerative treatment of intrabony defects: a novel approach to limit morbidity. J Clin Periodontol 2007;34:87-93.

29 Cortellini P, Nieri M, Prato GP, et al. Single minimally invasive surgical technique with an enamel matrix derivative to treat multiple adjacent intra-bony defects: clinical outcomes and patient morbidity. J Clin Periodontol 2008;35:605-13.

30 Aimetti M, Ferrarotti F, Mariani GM, et al. A novel flapless approach versus minimally invasive surgery in periodontal regeneration with enamel matrix derivative proteins: a 24-month randomized controlled clinical trial. Clin Oral Investig 2017;21:327-37.

31 Liu S, Hu B, Zhang Y, et al. Minimally invasive surgery combined with regenerative biomaterials in treating intra-bony defects: a metaanalysis. PLoS One 2016;11:e0147001.

32 Palachur D, Prabhakara Rao KV, Murthy KRV, et al. A comparative evaluation of bovine-derived xenograft (Bio-Oss collagen) and type I collagen membrane (Bio-Gide) with bovine-derived xenograft (BioOss collagen) and fibrin fibronectin sealing system (TISSEEL) in the treatment of intrabony defects: a clinico-radiographic study. J Indian Soc Periodontol 2014;18:336-43.

33 Sculean A, Chiantella GC, Windisch P, et al. Healing of intra-bony defects following treatment with a composite bovine-derived xenograft (Bio-Oss collagen) in combination with a collagen membrane (Bio-Gide PERIO). J Clin Periodontol 2005;32:720-4.
34 Tonetti MS, Greenwell H, Kornman KS. Staging and grading of periodontitis: framework and proposal of a new classification and case definition. J Clin Periodontol 2018;45:S149-61.

35 De Bruyckere T, Eghbali A, Younes F, et al. A 5-year prospective study on regenerative periodontal therapy of infrabony defects using minimally invasive surgery and a collagen-enriched bovine-derived xenograft. Clin Oral Investig 2018;22:1235-42.

36 Cosyn J, Cleymaet R, Hanselaer L, et al. Regenerative periodontal therapy of infrabony defects using minimally invasive surgery and a collagen-enriched bovine-derived xenograft: a 1-year prospective study on clinical and aesthetic outcome. J Clin Periodontol 2012;39:979-86.

37 Cortellini P, Tonetti MS. Clinical and radiographic outcomes of the modified minimally invasive surgical technique with and without regenerative materials: a randomized-controlled trial in intra-bony defects. J Clin Periodontol 2011;38:365-73.

38 Mishra A, Avula H, Pathakota KR, et al. Efficacy of modified minimally invasive surgical technique in the treatment of human intrabony defects with or without use of rhPDGF-BB gel: a randomized controlled trial. J Clin Periodontol 2013;40:172-9.

39 Tonetti MS, Lang NP, Cortellini P, et al. Enamel matrix proteins in the regenerative therapy of deep intrabony defects. J Clin Periodontol 2002;29:317-25. 